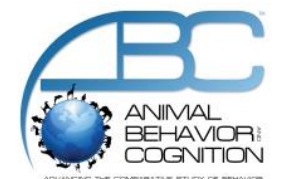

\title{
Behavior Systems Approach to Object Play: Stone Handling Repertoire as a Measure of Propensity for Complex Foraging and Percussive Tool Use in the Genus Macaca
}

\author{
Amanda N. Pelletier*1, Tatjana Kaufmann ${ }^{1}$, Sidhesh Mohak ${ }^{1}$, Riane Milan ${ }^{1}$, Charmalie A. D. \\ Nahallage $^{2}$, Michael A. Huffman ${ }^{3}$, Noëlle Gunst ${ }^{1}$, Aida Rompis ${ }^{4}$, I Nengah Wandia ${ }^{4}$, I Gusti Agung \\ Arta Putra $^{4}$, Sergio M. Pellis ${ }^{5}$, and Jean-Baptiste Leca ${ }^{1}$ \\ ${ }^{1}$ Department of Psychology, University of Lethbridge, Lethbridge, AB, Canada \\ ${ }^{2}$ Department of Sociology and Anthropology, University of Sri Jayewardenepura, Nugegoda, Sri Lanka \\ ${ }^{3}$ Department of Ecology and Social Behavior, Primate Research Institute, Kyoto University, Japan \\ ${ }^{4}$ Primate Research Center, Udayana University, Denpasar, Bali, Indonesia \\ ${ }^{5}$ Department of Neuroscience, University of Lethbridge, Lethbridge, AB, Canada \\ *Corresponding author (Email: Amanda.pelletier@uleth.ca)
}

Citation - Pelletier, A.N., Kaufmann, T., Mohak, S., Milan, R., Nahallage, C.A.D., Huffman, M.A., Gunst, N. Rompis, A., Wandia, I.N., Arta Putra, I.G.A., Pellis, S.M., \& Leca, J-B. (2017). Behavior systems approach to object play: Stone handling repertoire as a measure of propensity for complex foraging and percussive tool use in the genus Macaca. Animal Behavior and Cognition, 4(4), 455-473. https://dx.doi.org/10.26451/abc.04.04.05.2017

\begin{abstract}
Stone handling (SH), has been identified in four closely related primate species of the Macaca genus. We provide the first ethogram of SH in long-tailed macaques (Macaca fascicularis), a primate species known to use stones for extractive foraging. A total of $62.7 \mathrm{hrs}$ of video recorded data were scored from a population of Balinese long-tailed macaques living in Ubud, Bali, Indonesia, and a total of 36 stone handling patterns were identified. Behavior discovery curves were generated and showed that the minimum threshold of completeness was exceeded for the SH repertoire in this group. A "foraging substitute" hypothesis for the expression of SH was proposed, suggesting that $\mathrm{SH}$ consists of performing foraging-like actions on non-edible objects. We used a "behavior systems" framework to test this prediction, finding that all 36 stone handling patterns could be reliably categorized in a foraging behavior system, supporting the hypothesis that stone handling can be considered pseudo-foraging behavior. Our "behavior systems" approach will serve as a foundation for the future testing of the motivational basis of stone handling. Additionally, a comparison of 39 stone handling patterns performed by three macaque species $(M$. fascicularis, M. fuscata and M. mulatta) showed overlapping behavioral propensities to manipulate stones; however, the differences suggest that long-tailed macaques might be more prone to use stones as percussive tools in a foraging context. This work could offer insights into the development and evolution of complex activities such as percussive stone tool use in early humans.
\end{abstract}

Keywords - Object play, Percussive tool use, Behavior systems, Motivation, Foraging, Ethogram

"What we need are more detailed observations of play from the viewpoint of the various systems involved within what seems to be a single kind of play ..." (Burghardt, 2005, p. 137). Despite the lack of a definitional and theoretical consensus, object-oriented play is known to occur in numerous mammal and bird species (Fagen, 1981; Power, 2000). For the purpose of this study, object play will be defined as the spontaneous, repeated, seemingly relaxed, incompletely functional, and usually solitary manipulation of 
inanimate objects, which differs structurally, sequentially, and contextually from more serious versions of object handling (Bjorklund \& Pellegrini, 2002; Burghardt, 2005; Hall, 1998). Research on object play behavior in non-human primates has key implications for the development of foraging competence, the motivation underlying tool use, and the evolution of material culture in humans (Parker \& Gibson, 1977; Ramsey \& McGrew, 2005). The development of ethograms across a wider range of species is an important first step to better understanding object play. Because object play is rarely observed in adult animals - with the exception of some domestic species and captive individuals (Hall, 1998) - more detailed descriptions of adult animals playing with non-edible objects are also needed. Such data would aid in determining what actions are specific to play and which actions arise from immaturity when performed by young animals.

Stone handling $(\mathrm{SH})$ is one of the few types of object play routinely performed throughout an individual's lifespan in both captive and free-ranging groups, and has been described in four closely related species of macaques: Japanese macaques (Macaca fuscata), rhesus macaques (M. mulatta), longtailed macaques (M. fascicularis), and Taiwanese macaques (M. cyclopis, Nahallage, Leca, \& Huffman, 2016). SH consists of the non-instrumental manipulation of stones in various ways (e.g., clacking two stones together or rubbing stones on a substrate), and is structurally complex: the stones may be manipulated in combination with other objects (including edible ones, like fruits and leaves) and may involve various other body parts than just the hands (e.g., feet, mouth; Leca, Gunst, \& Huffman, 2008). In free-ranging provisioned groups of Japanese macaques, SH has been motivationally linked to foraging due to the similarities in the actions performed and the temporal association between these two objectoriented activities (Leca et al., 2008a). Additionally, SH is probably the best-known example of nonadaptive and culturally-transmitted behavior in non-human primates (Leca, Gunst, \& Huffman, 2012). Overall, $\mathrm{SH}$ is an ideal candidate behavior to examine the motivational processes underlying object play from a cross-species comparative perspective.

The first objective of this study was to contribute to the limited descriptive database of object play activities in both young and adult monkeys by providing a comprehensive written and videoillustrated SH ethogram in long-tailed macaques. The long-tailed macaque is an excellent candidate species to make this contribution, as one of the subspecies (i.e., Burmese long-tailed macaque, Macaca fascicularis aurea) is known for its manual dexterity and routine stone tool use skills in an extractive foraging context (Gumert, Kluck, \& Malaivijitnond, 2009).

Our second objective was to propose a "foraging substitute" hypothesis for the motivation underlying the expression of $\mathrm{SH}$ in this species. In line with previous research on Japanese macaques (Leca et al., 2008a), we suggest that SH consists of performing foraging-like actions on non-edible objects (i.e., stones) because this activity involves motivational processes typically associated with foraging. To explore this hypothesis, we used a "behavior systems" approach. This approach allows for the descriptive grouping of activity-specific behavioral patterns under multi-level, interrelated and hierarchically nested perceptual, central, and motor units, by inferring stimulus processing modules and more integrated internal states or motivational modes, which originate from a few major functional behavior systems (e.g., foraging, defense, sex/reproduction, parental care, socializing, and body care; Timberlake, 2001).

Our third objective was to compare the SH patterns performed by long-tailed macaques with the SH ethograms available for Japanese macaques (Leca, Gunst, \& Huffman, 2007) and rhesus macaques (Nahallage \& Huffman, 2008). Cross-species comparative analysis is one of the most powerful methodological tools to explore the origins and evolution of biological features (Martins, 1996). This approach is particularly useful to reconstruct scenarios for the evolutionary history of behavioral traits, which do not leave any direct fossil traces. It can be used to decide whether similar behavioral patterns are due to common ancestry or the result of independent adaptations to similar environmental pressures (Martins, 1996). Such a comparative approach should be relevant to understanding the evolution of object play behavior because it can distinguish adaptive from non-adaptive traits by indicating which ones have predated, accompanied, or followed the modification of some of their structural and functional attributes. The lack of functional constraints, and thus the flexibility and versatility of SH activity, makes it a good 
candidate for cross-species comparative analysis. If SH is most reliably assigned to the foraging behavior system in the long-tailed macaques, and the SH ethograms vary across these three macaque species, one could test whether this variation in object play behavior reflects inter-specific differences in foraging strategies. We put a special emphasis on the cross-species comparison of percussive SH patterns. Indeed, a higher diversity in percussive SH patterns and more frequent percussive stone-tool using in long-tailed macaques than in the other two macaque species could indicate differential adaptive foraging styles in relation to the behavioral propensity to manipulate stones.

\section{Methods}

\section{Study Site}

The study site was located at the Sacred Monkey Forest Sanctuary, Ubud (central Bali, Indonesia). The study population was composed of five neighboring groups of Balinese long-tailed macaques (Macaca fascicularis fascicularis), totaling approximately 600 individuals. In this study, we focused on one group (called "Cemetery"), totaling 136 individuals. The monkeys were free-ranging within the temple grounds and provisioned with fruits and vegetables by the temple staff twice daily.

This research was exclusively observational and non-invasive, and followed all Indonesian laws for foreign research. Our study was conducted in accordance with the Indonesian Ministry of Research and Technology, the Provincial Government of Bali, and the local district authorities, and approved by our federally mandated institutional animal welfare committee.

\section{Data Collection Procedure}

During three weeks in August 2008, between 09:00 hours and 18:00 hours, CADN, MAH and JBL used two main observational sampling methods: continuous focal-animal sampling and ad libitum sampling (Altmann, 1974). All focal and ad libitum samples were video-recorded with Sony digital video cameras (DCR-TRV22 and DCR-TRV33). Overall conditions of visibility were ideal for obtaining good quality video. Whenever possible, the subjects were filmed from the front or side, within $3-5 \mathrm{~m}$, and about $2 \mathrm{~m}$ square in frame. A total of $62.7 \mathrm{hrs}$ (i.e., $55.9 \mathrm{hrs}$ of focal and $6.8 \mathrm{hrs}$ of ad libitum samples) were collected from a representative subset of the population including all age and sex classes (namely, male and female infants, juveniles, and adults). Focal subjects were randomly selected, independently of their activities, and the age and sex class with least cumulative data was given priority. We followed Huffman's (1996) protocol, which previously determined that the optimal time period to record a complete SH bout of a randomly selected individual after feeding time was $15 \mathrm{~min}$. If the focal individual performed SH activity during the last 2 min of this $15 \mathrm{~min}$ period, the observation was extended for another 5 minutes before ending, unless $\mathrm{SH}$ was still in progress.

\section{Data Analysis}

In 2016, ANP used The Observer XT 12 (by Noldus) software to score the start and end time of each SH pattern down to the second, from a total of $14.0 \mathrm{hrs}$ of video-recorded SH activity. For the quantitative analyses of Balinese long-tailed macaques presented in this study, we used a sample of 7.3 hrs of SH performed by 75 individuals from all age/sex classes, which represented $205 \mathrm{SH}$ bouts. The basic unit of analysis that we employed to establish the SH ethogram was the SH pattern: a single, noninstrumental, stone-directed, and specifically defined manipulative action (see Appendix for a comprehensive list of distinct SH patterns in Balinese long-tailed macaques). We used The Observer XT 12 duration-sequence option to assess intra-scorer reliability for ANP when transcribing the same samples of SH video-records twice, involving a total of 15.6 min of video containing SH activity, with a total of 78 SH patterns performed ( $k=0.99$; Martin \& Bateson, 1993). 
To visually demonstrate the rate at which new SH patterns were identified over the course of observation time, we generated two behavior discovery curves - one generated from our focal sample and one from our ad libitum sample - by mapping the cumulative corresponding timecode (x-axis) at which all SH patterns first appeared (y-axis), using the order in which the videos were originally scored (Figures 1 and 2). In order to assess the completeness of the behavioral repertoire of SH patterns, we created an asymptotic model describing the relationship between the sampling effort and the observation of new SH patterns via a derivation of the Clench equation: $S(t)=a t /(1+b t)$ (Soberón \& Llorente, 1993), where $t$ is a measure of effort, $a$ is the rate of increase at the beginning of sampling, and $b$ is the accumulation of behavioral acts. After adjusting this equation to our data, we estimated the maximum theoretical number of SH patterns in our study group by calculating $\mathrm{a} / \mathrm{b}$ and the proportion of observed $\mathrm{SH}$ patterns (Dias, Rangel-Negrín, Covohua-Fuentes, \& Canales-Espinosa, 2009).

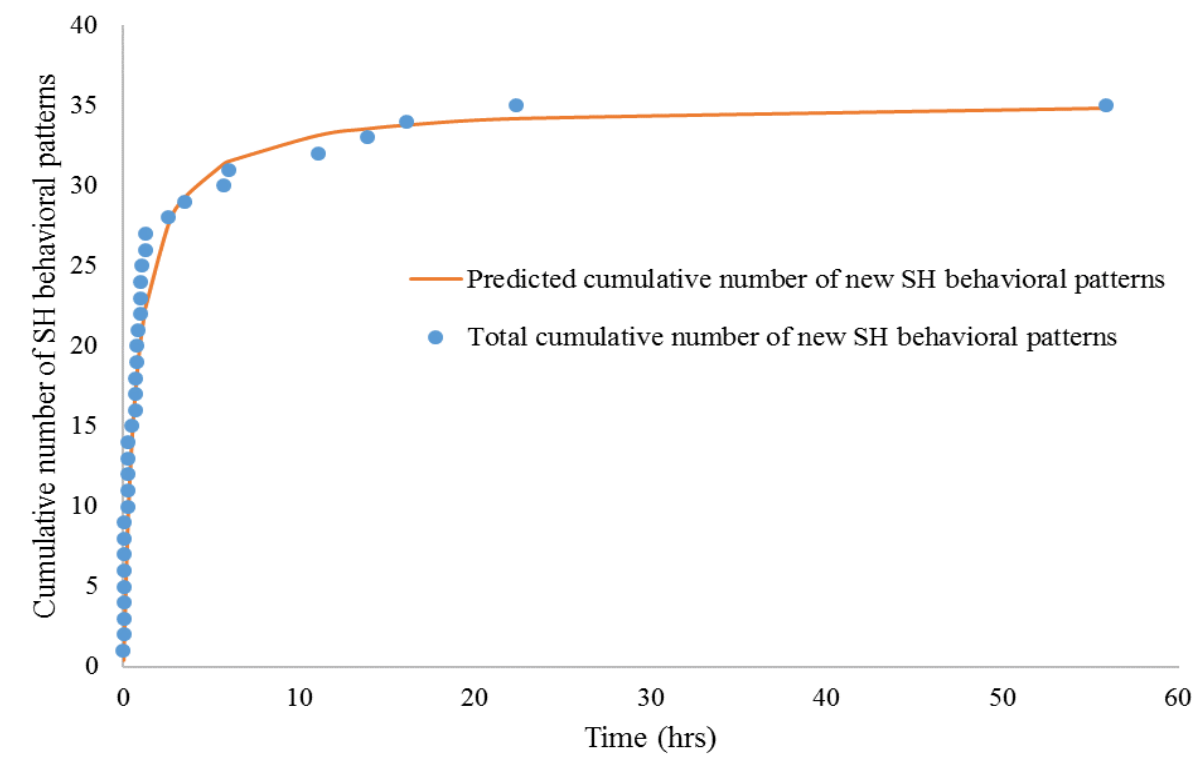

Figure 1. Behavior discovery curve representing the observed and predicted cumulative numbers of SH patterns obtained from focal data as a function of observation time.

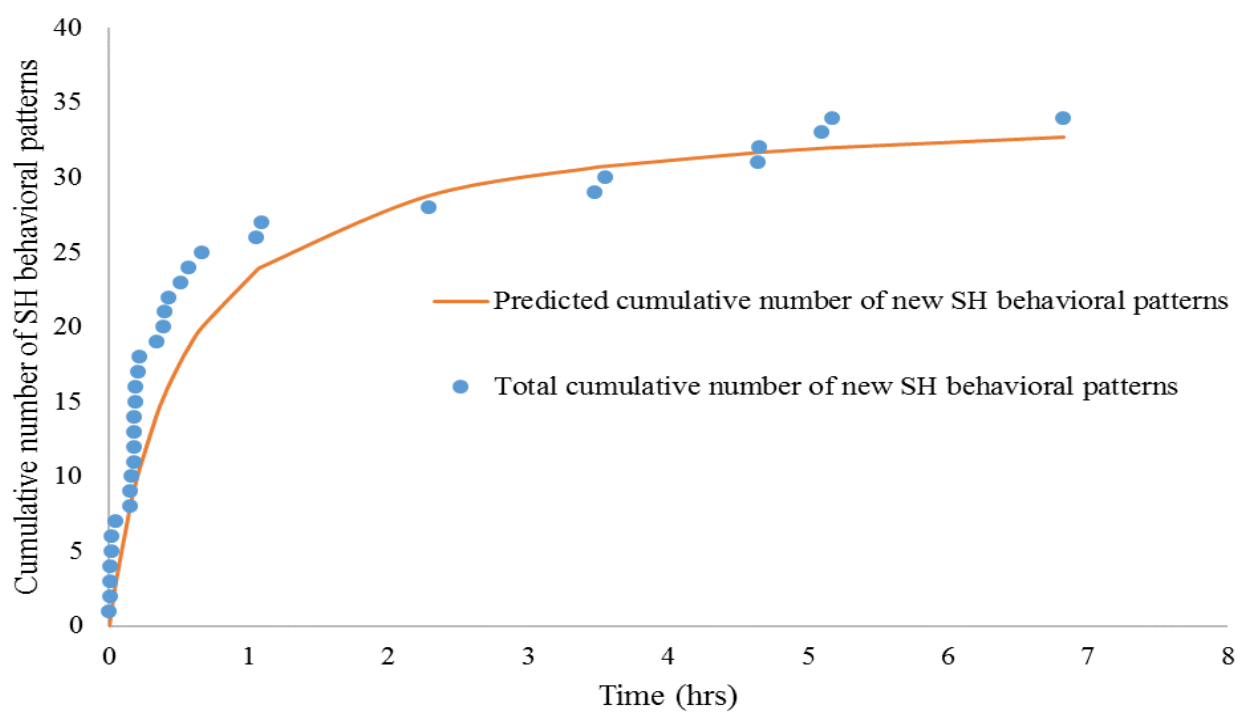

Figure 2. Behavior discovery curve representing the observed and predicted cumulative numbers of SH patterns obtained from ad libitum data, as a function of observation time. 
To reduce the possible effect of behavioral idiosyncrasy on the SH behavior discovery curve, we sampled a large number of individuals performing $\mathrm{SH}$, from all age and sex classes. Indeed, a previous study aiming to establish the ethogram of red pandas (Ailurus fulgens fulgens) and using a similar model showed that, if the total number of observation hours exceeds the number of animals observed, and if the degree of behavioral idiosyncrasy in the population is relatively low, the number of behaviors observed per hour increases at a more rapid rate when observing several animals within an observation time than when observing one single animal for the same length of time. In other words, it is better to observe more individuals in any given observation period than one individual for a long period of time (Jule, Lea, \& Leaver, 2009). Our study met these two requirements. First, 73 individuals (i.e., 97\% of identified stone handlers in the Cemetery group) were sampled over 55.9 hrs to generate the curve based on focal data, and 22 individuals (i.e., $29 \%$ of identified stone handlers) were sampled over $6.8 \mathrm{hrs}$ to generate the curve based on ad libitum data (Figure 2). Second, we aimed to establish the ethogram of SH, a cultural behavior with relatively low levels of idiosyncrasy ( $c f$. Leca et al., 2007).

To conduct a cross-species comparison of SH with the genus Macaca, we used previously published data on the SH behavior in Japanese macaques and rhesus macaques. The first data set was collected in 2004, in the free-ranging provisioned group of Japanese macaques, totaling 141 individuals living at the Iwatayama Monkey Park, Arashiyama, Kyoto Prefecture, Japan (cf. Leca et al., 2007). For the quantitative analyses of Japanese macaques presented in this study, we used a sample of 7.1 hours of SH performed by 63 individuals from all age/sex classes, which represented 149 SH bouts. The second data set was collected in 2004, in the captive group of rhesus macaques, totaling 29 individuals housed in an outside enclosure at the Kyoto University Primate Research Institute, Inuyama, Japan (cf. Nahallage \& Huffman, 2008). For the quantitative analyses of rhesus macaques presented in this study, we used a sample of $5.2 \mathrm{hrs}$ of SH performed by 29 individuals from all age/sex classes, which represented $103 \mathrm{SH}$ bouts.

To investigate possible differences in the occurrence (i.e., presence/absence) of each of the $39 \mathrm{SH}$ patterns (i.e., dichotomous nominal data) across three macaque species (namely, M. fascicularis, $M$. mulatta, and M. fuscata), we used a Cochran's Q test, followed by a series of post-hoc paired McNemar's tests. The qualitative comparison of percussive SH patterns (namely, Clack, Flint, Pound, Pound-Drag, Slap, Slap-Roll, Swipe, and Tap; Appendix) was based on the occurrence of these eight SH patterns across the three macaque species. The quantitative comparison of percussive SH patterns could be done only between $M$. fascicularis and M. fuscata. To compare the relative frequency of percussive SH patterns (i.e., how often percussive SH patterns were performed in a given SH bout, relative to other SH patterns) and the relative duration of percussive $\mathrm{SH}$ patterns (i.e., how long percussive $\mathrm{SH}$ patterns were performed in a given $\mathrm{SH}$ bout, relative to other SH patterns) between the two species, we used Mann-Whitney U tests. To compare the prevalence of percussive SH (i.e., how many group members engaged in this form of object play) between the two species, we used a 2 × 2 contingency chi-square test. For statistical analyses, we used IBM SPSS Statistics 24@. Because none of our predictions were directional, we conducted two-tailed tests. Significance levels were set at $\alpha=0.05$.

\section{Results}

\section{SH Ethogram}

The SH ethogram of the Balinese long-tailed macaques of Ubud included 36 behavioral patterns (Appendix; Figure 3). Descriptions and comments were included for each SH pattern. For clarity and simplicity, descriptions defined the actions as if they were performed using only one stone, even though most SH patterns could be performed using more than one stone. Corresponding videos were included in supplementary materials. The SH ethogram is available at: https://youtu.be/oRDvBbywJus. Examples of SH sequences are available at: https://youtu.be/MvvSg5Jo3JE 


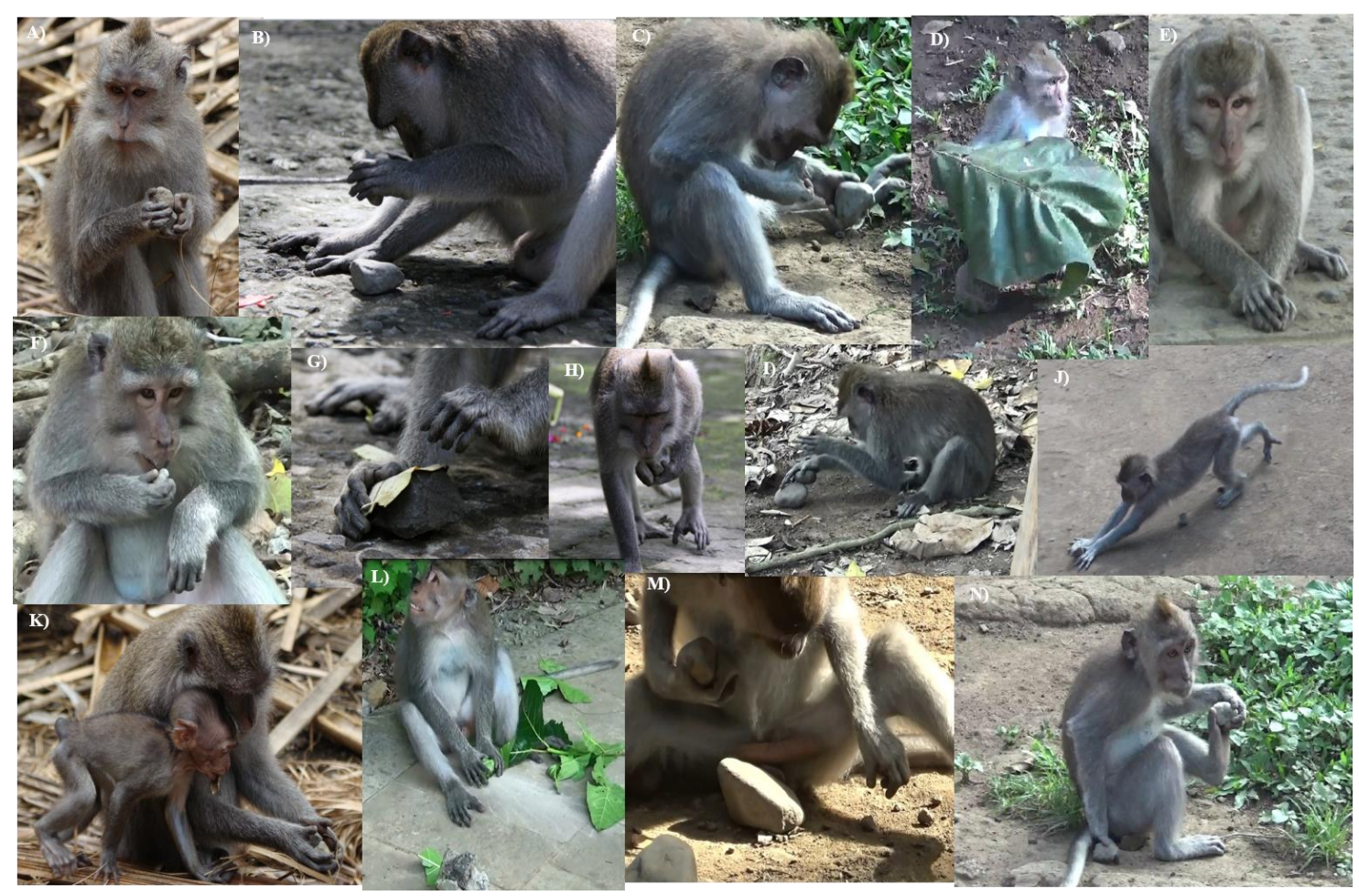

Figure 3. Examples of stone handling (SH) patterns by Balinese long-tailed macaques at Ubud, Bali, Indonesia. (A) Roll In Hands, (B) Pound, (C) Sniff, (D) Cover, (E) Roll, (F) Flint (in mouth), (G) Grasp, (H) Carry, (I) Gather, (J) Move And Push, (K) Rub Together, (L) Wrap, (M) Cuddle, (N) Hold. (Photos by J.-B. Leca).

To assess the completeness of the SH ethogram, we fit the observed cumulative number of newly scored SH patterns over the course of observation time with the predicted discovery curve for both focal samples (Figure 1) and ad libitum samples (Figure 2). The fit of our data to the Clench equation was very good for both the focal data $\left(r^{2} \geq 0.96\right)$ and the ad libitum data $\left(r^{2} \geq 0.98\right)$. Most SH patterns were discovered within the first hour of video recording for both sampling methods and the number of new behavioral patterns discovered decreased with more hours of observation. We found 35 behavioral patterns from the focal data, which closely matched $(99.3 \%)$ the expected theoretical number of $35.3(\mathrm{a}=$ $49.448, \mathrm{~b}=1.402)$. Likewise, we found 34 behavioral patterns from the ad libitum data, also closely matching $(96.8 \%)$ the expected theoretical number of $35.1(\mathrm{a}=70.139, \mathrm{~b}=1.997)$. These two curves revealed a plateau at 22.3 and $5.2 \mathrm{hrs}$ of observation, respectively. Overall, we exceeded the minimum threshold of completeness for the SH ethogram in this group (i.e., 90\%; Dias et al., 2009). Thus, the behavior discovery curves (Figure 1 and Figure 2) provide evidence that this comprehensive ethogram is representative of the SH patterns performed in the Ubud population of long-tailed macaques in 2008.

\section{"Behavior Systems" Approach to SH}

In order to explore the motivational processes underlying the SH activity, we utilized a "behavior systems" approach (Timberlake, 2001). We provided the best possible descriptive correspondence between each of the $36 \mathrm{SH}$ behavioral patterns and upper-level perceptual and central units from the most likely behavior system that has been previously associated with $\mathrm{SH}$ in macaques, namely the foraging behavior system (Huffman \& Quiatt, 1986; Leca et al., 2008a; Figure 4). Based on the behavioral repertoire of the long-tailed macaques (Brotcorne, 2014), we found that all $36 \mathrm{SH}$ patterns could most reliably be categorized in a nested and hierarchically organized foraging behavior system, including all the typical motivational modes characteristic of the chronological sequence of this activity, namely food search, food investigation, food processing, food extraction, and food consumption (Figure 4). 
Pelletier et al. 461

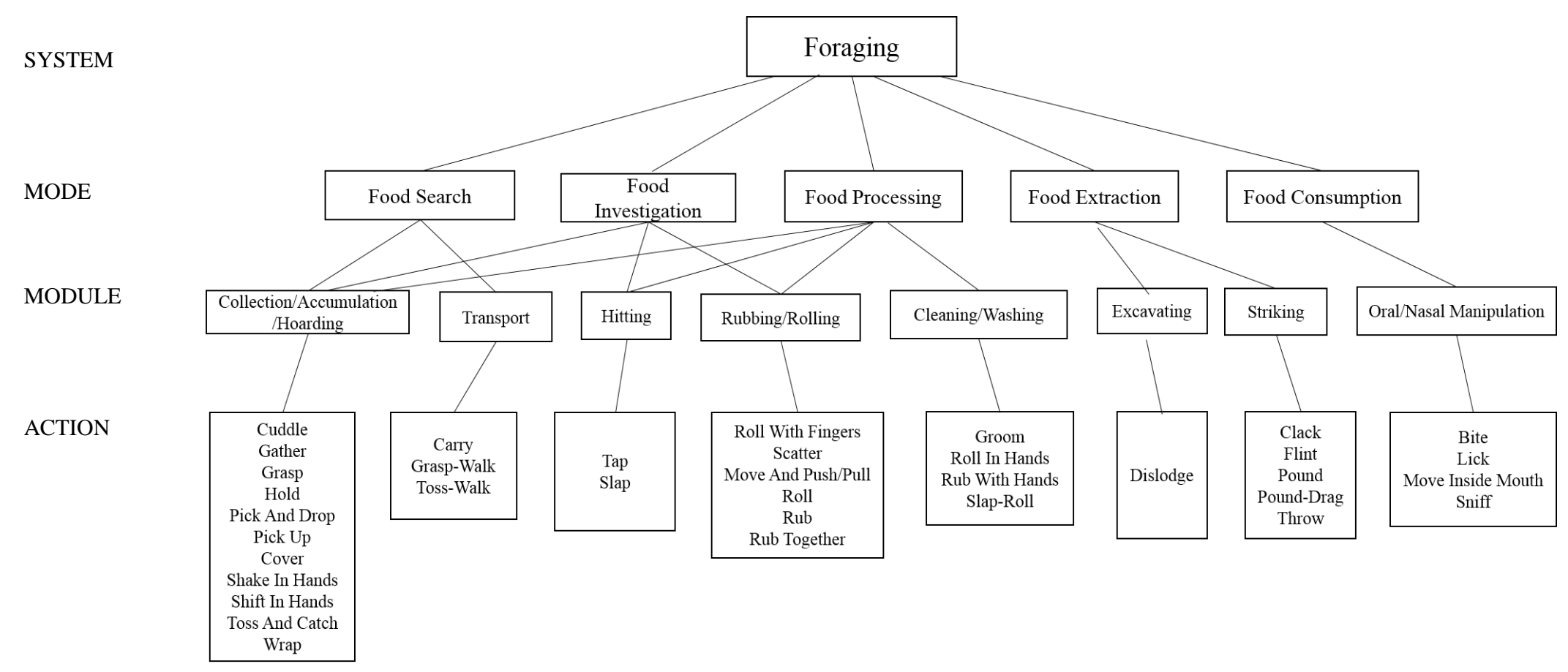

Figure 4. Behavior systems diagram representing the foraging behavior system in which the $36 \mathrm{SH}$ patterns displayed by Balinese long-tailed macaques can most reliably be grouped. 


\section{Cross-Species Comparative Analysis of SH, with an Emphasis on Percussive SH}

The patterns of SH performed by long-tailed macaques were compared to those previously described in Japanese macaques (Leca et al., 2007) and rhesus macaques (Nahallage \& Huffman, 2008). Previous classifications of SH patterns in both Japanese macaques and rhesus macaques were categorized based on general activity patterns and the combination with other objects (Leca et al., 2007; Nahallage \& Huffman, 2008). For this comprehensive SH ethogram in long-tailed macaques, SH patterns were specifically examined in relation to the precise movement being performed by the body parts executing the action (i.e., the hands or feet), rather than classifying SH patterns solely on the objects and body parts involved. Because long-tailed macaques often incorporate various objects and body parts into their SH activities (e.g., "Tap" on foot, groin, leg, tail; Pelletier, Huffman, Nahallage, Gunst, \& Leca, 2016), this method allowed us to create a smaller but more precise repertoire that labeled the behavioral patterns as general categories rather than splitting them when the general action being performed was the same (e.g., "Rub" includes patterns that involve a stone being moved back and forth along a substrate, and contains the previously labeled patterns: "Rub in mouth," "Rub with mouth," "Rub/put on fur," and "Stonegroom").

Table 1 shows how the previously labeled SH patterns ( $c f$. Leca et al., 2007; Nahallage \& Huffman, 2008) not included in this ethogram have now been categorized. Previous patterns such as "Insert into cavity," "Wash," "Combine with objects," and "Flint in mouth," were represented under broader categories based on the specific actions being performed by the hands or feet. These SH patterns were also present in long-tailed macaques; however, because our study group lives in a highly anthropogenic environment (Brotcorne, 2014), these monkeys have been observed to perform numerous SH patterns in combination with a variety of objects other than stones, including vegetal materials and human-made items (e.g., "Cover" with leaves, grass, cloth; "Pound" on leaf, nut, plastic; Pelletier et al., 2016), the previous method of classification would have led to a long and potentially confusing ethogram (e.g., "Cover with leaf," "Cover with cloth," "Cover with grass," "Cover with plastic"). This systematic method of classification prevented us from generating an ethogram that would have been either too general (e.g., "Combine with object" which could contain different actions such as "Cover" or "Wrap"), too specific (e.g., "Wrap with leaf," "Wrap with plastic"), or based on environmental factors including the objects or substrates involved (e.g., "Wash" and "Rub With Hands" were patterns in which the actions performed are the same, only the presence of water distinguishes them from one another). Because inserting stones into a cavity could also be viewed as the previously categorized pattern, "Combine with objects" (e.g., a bamboo stalk used as the cavity the stones are being inserted into and removed from), and because this action can be performed in a number of different ways by both the same individual, and between individuals, this previously identified category has now been categorized according to how the action is being performed (i.e., "Insert into cavity" frequently resembles a combination of the actions "Pick and drop" when the stone is dropped into a bamboo stalk, and "Gather" when the stone is then retrieved).

From this new categorization, a list of 39 distinct $\mathrm{SH}$ patterns was generated. Of the $39 \mathrm{SH}$ patterns, 36 were present in long-tailed macaques, 31 in Japanese macaques and 17 in rhesus macaques (Table 1). The occurrence of these $39 \mathrm{SH}$ patterns significantly differed across the three macaque species (Cochran's $Q(2)=26.4, p<0.001)$. Post-hoc paired comparisons showed significant differences in the SH repertoires of M. mulatta and M. fascicularis (McNemar's test, $p<0.001$ ), and that of $M$. mulatta and $M$. fuscata ( $p<0.001)$. The size of the SH repertoire of $M$. mulatta was about half as large as that of $M$. fascicularis and M. fuscata, and there was no SH pattern that was present in M. mulatta and absent in $M$. fascicularis or $M$. fuscata. The SH repertoires of $M$. fascicularis and $M$. fuscata did not differ significantly in profile (McNemar's test, $p=0.227$ ), nor in size (Table 1).

Long-tailed macaques showed a higher diversity in percussive SH patterns than Japanese and rhesus macaques. Among the eight percussive SH patterns documented in the genus Macaca to date, $M$. fascicularis performed seven (namely, Clack, Flint, Pound, Pound-Drag, Slap, Slap-Roll, and Tap), $M$. fuscata three (Flint, Pound, and Swipe), and M. mulatta one (Clack; Appendix). Even though $M$. 
fascicularis and M. fuscata did not differ significantly in the duration of SH bouts (mean $\pm \mathrm{SD}, 2.2 \pm 3.2$ min and $2.9 \pm 3.7 \mathrm{~min}$, respectively; Mann-Whitney U test, $U=13405, p=0.050$ ), percussive SH patterns were significantly more frequent $(U=7199, p<0.001)$ and lasted significantly longer $(U=7146, p<$ 0.001 ) in $M$. fascicularis than in M. fuscata. Although $M$. fascicularis and $M$. fuscata did not differ significantly in the prevalence of SH activity (55.1\% and $44.7 \%$ of sampled individuals performed $\mathrm{SH}$, respectively; $\left.\chi^{2}(1)=3.03, p=0.082\right)$, the prevalence of percussive SH patterns was significantly higher in M. fascicularis than in M. fuscata $(74.7 \%$ and $4.8 \%$ of sampled stone handlers performed percussive $\mathrm{SH}$, respectively; $\left.\chi^{2}(1)=68.36, p<0.001\right)$.

Table 1

Presence (X) or Absence (-) of 39 SH Behavioral Patterns, Based on Previously Published and Slightly Modified Classifications (cf. Last Column on the Far Right) of SH Patterns Across Three Species in the Macaca Genus (for M. mulatta, see Nahallage \& Huffman, 2008; for M. fuscata, see Leca et al., 2007).

\begin{tabular}{|c|c|c|c|c|}
\hline $\begin{array}{c}\text { SH Behavioral } \\
\text { Pattern }\end{array}$ & $\begin{array}{c}\text { Macaca } \\
\text { fascicularis }\end{array}$ & $\begin{array}{l}\text { Macaca } \\
\text { mulatta }\end{array}$ & $\begin{array}{l}\text { Macaca } \\
\text { fuscata }\end{array}$ & Previous Classification of SH Patterns \\
\hline Bite & $\mathrm{X}$ & $\mathrm{X}$ & $\mathrm{X}$ & \\
\hline Carry & $\mathrm{X}$ & $\mathrm{X}$ & $\mathrm{X}$ & Carry in mouth \\
\hline Clack & $X$ & $X$ & $X$ & \\
\hline Cover & $X$ & - & $\mathrm{X}$ & $\begin{array}{l}\text { Wrap in leaf was not previously distinguished from Cover, } \\
\text { Combine with objects }\end{array}$ \\
\hline Cuddle & $\mathrm{X}$ & $\mathrm{X}$ & $X$ & \\
\hline Dislodge & $X$ & - & - & \\
\hline Flint & $\mathrm{X}$ & - & $X$ & Flint in mouth \\
\hline Flip* & - & - & $X$ & \\
\hline Gather & $\mathrm{X}$ & - & $\mathrm{X}$ & Pick, Put in water \\
\hline Grasp & $\mathrm{X}$ & - & $\mathrm{X}$ & Grasp with hands \\
\hline Grasp-Walk & $\mathrm{X}$ & $\mathrm{X}$ & $\mathrm{X}$ & \\
\hline Groom & $X$ & - & - & \\
\hline Hold & $\mathrm{X}$ & $\mathrm{X}$ & $\mathrm{X}$ & \\
\hline Lick & $\mathrm{X}$ & $\mathrm{X}$ & $\mathrm{X}$ & \\
\hline Move And Push/Pull & $\mathrm{X}$ & $\mathrm{X}$ & $\mathrm{X}$ & \\
\hline Move Inside Mouth & $X$ & $\mathrm{X}$ & $\mathrm{X}$ & Put in mouth \\
\hline Pick And Drop & $\mathrm{X}$ & $\mathrm{X}$ & $X$ & Pick up small stones, Insert into cavity \\
\hline Pick Up & $\mathrm{X}$ & - & $\mathrm{X}$ & \\
\hline Pound & $\mathrm{X}$ & - & $\mathrm{X}$ & \\
\hline Pound-Drag & $\mathrm{X}$ & - & - & \\
\hline Roll & $\mathrm{X}$ & $\mathrm{X}$ & $\mathrm{X}$ & Rub on surface was not previously distinguished from Roll \\
\hline Roll In Hands & $X$ & $X$ & $X$ & \\
\hline Roll With Fingers & $\mathrm{X}$ & - & - & \\
\hline Rub & $X$ & $X$ & $X$ & $\begin{array}{l}\text { Rub/put on fur, Stone-groom, Rub with mouth, Rub in } \\
\text { mouth }\end{array}$ \\
\hline Rub Together & $\mathrm{X}$ & $\mathrm{X}$ & $\mathrm{X}$ & \\
\hline Rub With Hands & $X$ & - & $X$ & Wash when performed in water \\
\hline Scatter & $\mathrm{X}$ & $\mathrm{X}$ & $\mathrm{X}$ & \\
\hline Shake In Hands & $\mathrm{X}$ & - & $\mathrm{X}$ & \\
\hline Shift In Hands & $\mathrm{X}$ & - & - & \\
\hline Slap & $\mathrm{X}$ & - & $\mathrm{X}$ & Tap in mouth \\
\hline Slap-Roll* & $\mathrm{X}$ & - & - & \\
\hline Sniff & $\mathrm{X}$ & $\mathrm{X}$ & $\mathrm{X}$ & \\
\hline Spin* & - & - & $X$ & \\
\hline Swipe* & - & - & $\mathrm{X}$ & \\
\hline Tap & $\mathrm{X}$ & - & - & \\
\hline Toss And Catch* & $X$ & - & - & \\
\hline Toss-Walk & $\mathrm{X}$ & $\mathrm{X}$ & $\mathrm{X}$ & \\
\hline Throw & $\mathrm{X}$ & - & $\mathrm{X}$ & Throw and jump, Throw and run, Throw and sway \\
\hline Wrap & $\mathrm{X}$ & - & $\mathrm{X}$ & Wrap in leaf, Combine with objects \\
\hline Total SH Patterns & 36 & 17 & 31 & \\
\hline
\end{tabular}


It is also noteworthy that percussive SH patterns were performed by numerous individuals from all age and sex classes in M. fascicularis. Of the 75 stone handlers sampled in this species, including 11 adult males, 23 adult females, 21 juvenile males, and 20 juvenile females, 58 individuals performed percussive SH patterns, including 10 adult males, 21 adult females 15 juvenile males, and 10 juvenile females. By contrast, of the 63 stone handlers sampled in $M$. fuscata, including eight adults males, 44 adult females, five juvenile males, and six juvenile females, only three individuals performed percussive SH patterns, including two adult females and one juvenile male.

\section{Discussion}

This paper provides the first $\mathrm{SH}$ ethogram in Balinese long-tailed macaques. Categorizing behavior by using descriptive observations is a first step towards understanding these motivations, as it provides a thorough foundation on which to base future sequential and kinematic analyses. We used a theoretical model (i.e., the behavior systems approach) to propose structural connections between $\mathrm{SH}$, an object play activity in this primate species, and more functional behavioral categories. By doing so, we inferred potential motivations for this playful and thus, incompletely functional behavior. Understanding the motivations underlying object play behavioral patterns, specifically $\mathrm{SH}$ actions that are performed throughout the lifespan, can offer insight into the fitness consequences of playful activities. Because $\mathrm{SH}$ is routinely performed by both young individuals and adults, health-related and welfare benefits of this behavior have been suggested (see Nahallage \& Huffman, 2007a; Nahallage et al., 2016).

Our preliminary behavior systems approach suggested that, while showing most characteristics of object play, $\mathrm{SH}$ might be considered a foraging-like activity that consists of pseudo-foraging behavioral patterns directed toward non-edible objects. First, Balinese long-tailed macaques were as much manipulative with stones as with food items that are difficult to process. Of the $36 \mathrm{SH}$ patterns exhibited by Balinese long-tailed macaques (i.e., all those listed in Table 1, except Rub Together, and Toss And Catch), 34 have also been observed being performed in an extractive foraging context: nut handling, a food-processing activity aimed at weakening the hard shell of three types of local fruits and nuts (i.e., Cocos nucifera, Aleurites moluccanus and Pangium edule) in order to crack them open and feed on the seeds inside (Pelletier et al., 2017). Second, we argued that a given SH pattern (i.e., Grasp) could be ascribed to the most relevant perceptual-motor modules (i.e., collection/accumulation/hoarding), which in turn, could be included in the most putative motivational modes (i.e., food search) within a specific behavior system (i.e., foraging). Third, SH could be viewed as the appetitive phase of the foraging behavior system, with obviously no consummatory phase, because the object being manipulated (i.e., a stone) is not edible. Fourth, in free-ranging groups of Japanese macaques, most SH activity occurred within 20 min following food provisioning time and while the monkeys were still chewing their food (Leca et al., 2008a). As such, our findings were consistent with the view that the structure of play behavior is amenable to a behavior systems approach (Pellegrini, 2009; Pellis \& Pellis, 2009). Object play behavior has also been motivationally linked to foraging activities in other mammals (oriental smallclawed otters: Pellis, 1991; domestic cats: Hall \& Bradshaw, 1998).

Of course, the involvement of other behavior systems is also possible. For example, we argued that the SH pattern "Throw" should primarily be ascribed to the foraging behavior system because it was reminiscent of the underhand throwing motion of a hard-shelled food item (e.g., coconut) up in the air to crack it open, an innovative foraging technique observed in several macaque species (e.g., rhesus macaques: Comins, Russ, Humbert, \& Hauser, 2011; Balinese long-tailed macaques: Gunst, personal observation). However, we acknowledge that a similar stone-directed upward throwing action could also fit into a defense behavior system (e.g., Japanese macaques: Leca, Nahallage, Gunst, \& Huffman, 2008). Still, we believe that our study provides a basis for the "foraging substitute" hypothesis that should be tested using a principal component analysis. If the SH patterns assigned to particular modules (e.g., Pick Up, Gather, Grasp) frequently co-occur within a given SH bout, it could suggest that their expression is indeed underlain by a unique and so-called "collection, accumulation, hoarding" motivational module under the "foraging behavior system" (Figure 4). 
Alternative functional hypotheses pertaining to the expression of SH have been tested in Japanese macaques, two of which received some support. In line with the "surplus energy" hypothesis, proposing that play behavior enables the adaptive expenditure of excess metabolic energy, SH bouts in juveniles (often not limited in energy) were more frequent, versatile, and vigorous, but shorter, than in adults (Leca et al., 2007). Consistent with the "motor training" hypothesis, SH could have beneficial consequences both in immature individuals by allowing a faster development of manipulative skills (Nahallage \& Huffman, 2007a) and in senescent individuals by maintaining neural pathways through the daily practice of fined-tuned manual activity, and potentially slowing down the deterioration of sensorimotor and cognitive abilities associated with advanced age (Nahallage et al., 2016). Additionally, even though the "misdirected foraging" hypothesis was not supported in a captive group of Japanese macaques (Nahallage \& Huffman, 2007a), it was supported in all free-ranging provisioned groups of this species, where there was a clear temporal connection between SH occurrence and the post-provisioning period (Leca et al., 2008a). Despite sometimes conflicting results, these alternative hypotheses are not mutually exclusive; they suggest that SH could be underlain by various motivational, cognitive, and maturational processes depending on the age class and the context in which this activity occurs. Future experimentally controlled studies should also examine whether the foraging behavior system approach to $\mathrm{SH}$ is causal in the context of free-ranging provisioned macaques. More specifically, individuals more frequently performing SH patterns that we ascribed to the food extraction module (i.e., "Clack," "Flint," "Pound") should be more likely to engage in experimentally-induced percussive stone tool use in a foraging context, due to their familiarity with stone-striking actions in a playful context.

A previous study of SH in Japanese and rhesus macaques showed some similarities in the SH patterns performed, suggesting a common behavioral propensity for $\mathrm{SH}$ in these two macaque species (Nahallage \& Huffman, 2008). Our SH ethogram in long-tailed macaques provides the basis for a comparison with a third species within the same genus. Table 1 showed only the presence or absence of SH patterns in these three species, and though this comparison should be viewed as preliminary, it is noteworthy that the SH profile of Balinese long-tailed macaques overlapped more with that of Japanese macaques than with that of rhesus macaques. Our results also showed that $\mathrm{SH}$ was significantly more diverse in Balinese long-tailed macaques and Japanese macaques than in rhesus macaques. With regards to percussive $\mathrm{SH}$, we found that percussive $\mathrm{SH}$ patterns were more diverse in Balinese long-tailed macaques than in Japanese and rhesus macaques. Percussive SH activity was also more prevalent, more frequent, and more enduring in Balinese long-tailed macaques than in Japanese macaques.

Even if our cross-species comparison is preliminary, these differences suggest that long-tailed macaques of all age and sex classes are more prone to use a variety of combinatorial and percussive actions during stone play activity than Japanese and rhesus macaques. Our results are consistent with a comparative analysis of object manipulation within the genus Macaca, and show that, among all four closely related macaque species exhibiting SH (i.e., M. fascicularis, $M$. fuscata, M. mulatta, and $M$. cyclopis; cf. Nahallage et al., 2016), long-tailed macaques displayed the greatest variety of finger use manipulation patterns (Torigoe, 1987). They are also consistent with a recent comparative analysis of manipulation complexity across 36 nonhuman primate species, using a scaling method with increasing complexity levels from 1 to 8 , based on the (a)synchronous (un)coordinated use of hands and digits with same/different objects; this study showed that Japanese macaques reached complexity level 6, whereas long-tailed macaques reached level 7 (Heldstab et al., 2016).

Interestingly, these findings on the playful manipulation of stones in Balinese long-tailed macaques parallel other stone-directed behavioral data collected in a more functional context in a closely related subspecies: Burmese long-tailed macaques are more frequent stone tool users than Japanese and rhesus macaques, and the only macaques spontaneously exhibiting percussive stone tool use techniques to crack shellfish in coastal environments (Gumert et al., 2009; Tan, 2016). Developmental evidence indicates that percussive stone tool use in Burmese long-tailed macaques may be facilitated by a biological predisposition to handle stones at a very young age, via exploratory and non-instrumental actions that are gradually incorporated into foraging routines (Tan, 2017). From an evolutionary viewpoint, our study suggests that cross-species variation in manual dexterity and the behavioral 
propensity to manipulate stones in a playful context could reflect, and possibly explain, differential adaptive foraging styles, including stone tool use, within the genus Macaca.

To further explore the behavior systems pertaining to $\mathrm{SH}$, one should test whether percussive $\mathrm{SH}$ and percussive stone tool use are underlain by similar motivational and cognitive processes. Future analyses will compare the kinematic structure of pounding actions with different objects in different contexts, as well as the temporal organization of different types of pounding sequences, in Balinese and Burmese long-tailed macaques. After controlling for age, and thus physical maturity, we expect higher structural complexity of pounding actions and more predictable behavioral sequences as the apparent functionality of the activity, the food-related interest in the objects being handled, and the difficulty to manipulate them increase. More specifically, we predict that among adults, the variability in the execution of arm/hand movements and the level of randomness in the behavioral sequences will decrease from nonpercussive SH (i.e., bouts including stone-gathering and stone-rubbing) to percussive SH (i.e., bouts including stone-pounding on the ground) to percussive food handling (i.e., bouts including nut-pounding on the ground) to stone tool use (i.e., bouts including stone-hammering on shellfish). This work could offer insights into the emergence of complex foraging activities such as percussive stone tool use in early humans.

Like other behavioral traditions (Fragaszy \& Perry, 2003), SH is socially learned, performed by most members of the group, transmitted over generations, and can be viewed as developing through numerous phases (Huffman, 1984; Huffman \& Quiatt, 1986; Huffman, 1996; Leca et al., 2012; Nahallage \& Huffman, 2007b). The beginning phase can be described as the innovation phase, where an individual performs a novel activity. The second phase, transmission, is the early part of the behavioral diffusion, and can be described as the diffusion of SH behavior from individuals through social means, typically horizontally among individuals in a close social network, such as playmates. In the later period of diffusion, when the behavior reaches the tradition phase, $\mathrm{SH}$ is passed down through generations, primarily from mothers to their offspring, and is performed by most members of the group (Huffman \& Hirata, 2003). An additional phase, the transformation phase, occurs after the behavior has reached a cultural level, and new patterns and modifications are added, leading to an increase in both repertoire size and complexity, and expanding the contexts in which they are performed (Huffman \& Quiatt, 1986; Leca et al., 2012). From this perspective, patterns such as "Slap-roll" and "Pound-drag" could be viewed as part of the transformation phase of $\mathrm{SH}$, as they may involve the combination of already established $\mathrm{SH}$ patterns to create new and more complex ones. Table 1 shows a total of eight new SH patterns identified in long-tailed macaques that have not been observed previously in other species. Patterns such as "Slaproll" and "Toss and Catch" were idiosyncratic, being very rare and performed by only one to three individuals, much like the "Flip," "Swipe," and "Spin" patterns in Japanese macaques, potentially speaking to the novelty or complexity of the patterns.

The transformation phase could also involve the combination of already established SH patterns with a variety of objects other than stones, body parts, and substrates. Patterns such as "Roll With Fingers," and different variants of "Throw" (i.e., "Throw and run," "Throw and jump," and "Throw and sway" performed in a group of Japanese macaques) are consistent with this view, as they involve previously established patterns being performed in a new way. Stone throwing in Japanese macaques is a perfect example of this. When a "Throw" is performed in combination with an agonistic display, it provides an effective signal to other individuals within the group, and can be considered a form of spontaneous tool use (Leca et al., 2008b). The "Tap" pattern in long-tailed macaques could also be viewed as part of the Transformation phase of SH as it also encompasses several different variants. This pattern can be performed using numerous body parts including tapping a stone onto the hands, feet, leg, tail, and groin, and often involves the combination with non-stone objects. Still, the primary focus on stones during the SH activity could be due to the fact that they are small, graspable, hard, soundproducing, multifunctional, and ubiquitous objects. As such, our research has implications for the evolution of combinatory object-directed actions, including stone tool use, in our primate ancestors. 


\section{Conclusion}

The lack of definitional and theoretical agreement regarding object play activities suggests that more detailed descriptions are needed. We presented the first SH ethogram in Balinese long-tailed macaques. The behavior systems approach provides a likely hypothesis for future research on the underlying motivations of SH. A systematic comparison of SH across multiple macaque species utilizing this framework could enhance our understanding of the development and evolution of complex manipulative activities in hominins, such as percussive stone tool use in a foraging context.

\section{Acknowledgements}

This study was funded by the following agencies: Japanese Ministry of Education, Science, Sports and Culture, Japan Society for the Promotion of Science (JSPS), HOPE Project, Natural Sciences and Engineering Research Council of Canada (NSERC), Japan Society for the Promotion of Science (JSPS), L.S.B. Leakey Foundation, and the Office of the Dean of Arts and Science and the Office of

Research Services at the University of Lethbridge. We thank two anonymous reviewers for fruitful comments on a previous version of the manuscript.

\section{References}

Altmann J. (1974). Observational study of behavior: Sampling methods. Behaviour, 49, 227-267.

Bjorklund, D. F., \& Pellegrini, A. D. (2002). The origins of human nature: Evolutionary developmental psychology. Washington, DC: American Psychological Association.

Brotcorne, F. (2014). Behavioral ecology of commensal long-tailed macaque (Macaca fascicularis) populations in Bali, Indonesia: Impact of anthropic factors. (Unpublished doctoral dissertation). University of Liège, Liège, Belgium.

Burghardt, G. M. (2005). The genesis of animal play: Testing the limits. Cambridge, MA: Bradford Books (MIT Press).

Comins, J. A., Russ, B. E., Humbert, K. A., \& Hauser, M. D. (2011). Innovative coconut-opening in a semi-free ranging rhesus monkey (Macaca mulatta): A case report on behavioral propensities. Journal of Ethology, $29,187-189$.

Dias, P. A. D., Rangel-Negrín, A., Coyohua-Fuentes, A., \& Canales-Espinosa, D. (2009). Behaviour accumulation curves: A method to study the completeness of behavioural repertoires. Animal Behaviour, 77, 1551-1553.

Fagen, R. (1981). Animal play behavior. New York: Oxford University Press.

Fragaszy, D., \& Perry, S. (2003). Towards a biology of traditions. In D. Fragaszy \& S. Perry (Eds.), The biology of traditions: Models and evidence (pp. 1-32). Cambridge, UK: Cambridge University Press.

Gumert, M. D., Kluck, M., \& Malaivijitnond, S. (2009). The physical characteristics and usage patterns of stone axe and pounding hammers used by long-tailed macaques in the Andaman sea region of Thailand. American Journal of Primatology, 71, 594-608.

Hall, S. (1998). Object play by adult animals. In M. Bekoff \& J. A. Byers (Eds.), Animal play: Evolutionary, comparative, and ecological perspectives (pp. 45-60). Cambridge, UK: Cambridge University Press.

Hall, S. L., \& Bradshaw, J. W. S. (1998). The influence of hunger on object play by adult domestic cats. Applied Animal Behaviour Science, 58, 143-150.

Heldstab, S. A., Kosonen, Z. K., Koski, S. E., Burkart, J. M., van Schaik, C. P., \& Karin Isler, K. (2016). Manipulation complexity in primates coevolved with brain size and terrestriality. Scientific Reports, 6, 24528.

Huffman, M. A. (1984). Stone-play of Macaca fuscata in Arashiyama B troop: Transmission of a non-adaptive behavior. Journal of Human Evolution, 13, 725-735.

Huffman, M. A. (1996). Acquisition of innovative cultural behaviors in non-human primates: A case study of stone handling, a socially transmitted behavior in Japanese macaques. In B. Galef, Jr. \& C. Heyes (Eds.), Social learning in animals: The roots of culture (pp. 267-289). Orlando, FL: Academic Press.

Huffman, M. A., \& Hirata, S. (2003). Biological and ecological foundations of primate behavioral tradition. In D. Fragaszy \& S. Perry (Eds.), The biology of tradition: Models and evidence (pp. 267-296). Cambridge, UK: Cambridge University Press. 
Huffman, M. A., \& Quiatt, D. (1986). Stone handling by Japanese macaques (Macaca fuscata): Implications for tool use of stone. Primates, 27, 413-423.

Jule, K. R., Lea, S. E. G., \& Leaver, L. A. (2009). Using a behaviour discovery curve to predict optimal observation time. Behaviour, 146, 1531-1542.

Leca, J.-B., Gunst, N., \& Huffman, M. A. (2007). Japanese macaque cultures: Inter- and intra-troop behavioural variability of stone handling patterns across 10 troops. Behaviour, 144, 251-281.

Leca, J.-B., Gunst, N., \& Huffman, M. A. (2008a). Food provisioning and stone handling tradition in Japanese macaques: A comparative study of ten troops. American Journal of Primatology, 70, 803-813.

Leca, J.-B., Nahallage, C. A. D., Gunst, N., Huffman, M. A. (2008b). Stone-throwing by Japanese macaques: Form and functional aspects of a group-specific behavioral tradition. Journal of Human Evolution, 55, 989-998.

Leca, J.-B., Gunst, N., \& Huffman, M. A. (2012). Thirty years of stone handling tradition in Arashiyama-Kyoto macaques: Implications for cumulative culture and tool use in non-human primates. In J.-B. Leca, M. A. Huffman, \& P. L. Vasey (Eds.), The monkeys of stormy mountain: 60 years of primatological research on the Japanese macaques of Arashiyama (pp. 223-257). Cambridge, UK: Cambridge University Press.

Martin, P., \& Bateson, P. (1993). Measuring behaviour: An introductory guide. Cambridge, UK: Cambridge University Press.

Martins, E. P. (1996). Phylogenies and the comparative method in animal behavior. New York, USA: Oxford University Press.

Nahallage, C. A. D., \& Huffman, M. A. (2007a). Age-specific functions of stone handling, a solitary-object play behavior, in Japanese macaques (Macaca fuscata). American Journal of Primatology, 69, 267-281.

Nahallage, C. A. D., \& Huffman, M. A. (2007b). Acquisition and development of stone handling behavior in infant Japanese macaques. Behaviour, 144, 1193-1215.

Nahallage, C. A. D., \& Huffman, M. A. (2008). Comparison of stone handling behaviour in two macaque species: Implications for the role of phylogeny and environment in primate cultural variation. American Journal of Primatology, 70, 1124-1132.

Nahallage, C. A. D., \& Huffman, M. A. (2012). Stone handling behavior in rhesus macaques (Macaca mulatta), a behavioral propensity for solitary object play shared with Japanese macaques. Primates, 53, 71-78.

Nahallage, C. A. D., Leca, J.-B., \& Huffman, M. A. (2016). Stone handling, an object play behaviour in macaques: Welfare and neurological health implications of a bio-culturally driven tradition. Behaviour, 153, 845-869.

Parker, S. T., \& Gibson, K. R. (1977). Object manipulation, tool use and sensorimotor intelligence as feeding adaptations in Cebus monkeys and great apes. Journal of Human Evolution, 6, 623-641.

Pellegrini, A. D. (2009). The role of play in human development. Oxford: Oxford University Press.

Pelletier, A. N., Huffman, M. A., Nahallage, C. A. D., Gunst, N., \& Leca, J.-B. (2016, February). Comparing stone play in two macaque species. Poster session presented at the Prairie University Biology Symposium, University of Lethbridge, Lethbridge, Canada.

Pelletier, A. N., Sita, S., Neugebauer, E., Gunst, N, Pellis, S. M., \& Leca, J.-B. (2017, June). Comparative analysis of two object-manipulation activities in Balinese long-tailed macaques. Paper session presented at the meeting of the Animal Behavior Society, University of Toronto Scarborough, Toronto, Canada.

Pellis, S. M. (1991). How motivationally distinct is play? A preliminary case study. Animal Behaviour, 42, 851-853.

Pellis, S. M., \& Pellis, V. C. (2009). The playful brain: Venturing to the limits of neuroscience. Oxford: Oneworld Press.

Power, T. G. (2000). Play and exploration in children and animals. Mahwah, NJ: Erlbaum.

Ramsey, J. K., \& McGrew, W. C. (2005). Object play in great apes: Studies in nature and captivity. In A. D. Pellegrini \& P. K. Smith (Eds.), The nature of play: Great apes and humans (pp. 89-112). New York: Guilford.

Soberón, J. M., \& Llorente, J. B. (1993). The use of species accumulation functions for the prediction of species richness. Conservation Biology, 7, 480-488.

Tan, A. W. Y. (2016). Behavioral processes and social influences on the development of stone-tool use in longtailed macaques. (Unpublished doctoral dissertation), Nanyang Technological University, Singapore.

Tan, A. W. Y. (2017). From play to proficiency: The ontogeny of stone-tool use in coastal-foraging long-tailed macaques (Macaca fascicularis) from a comparative perception-action perspective. Journal of Comparative Psychology, 131, 89-114.

Timberlake, W. (2001). Motivational modes in behavior systems. In R. R. Mowrer \& S. B. Klein (Eds.), Handbook of contemporary learning theories (pp. 155-210). San Francisco, CA: Erlbaum.

Torigoe, T. (1987). Further report on object manipulation in non-human primates: A comparison within 13 species of the genus Macaca. Primates, 28, 533-538. 


\section{Appendix}

Ethogram comprised of 36 stone handling patterns performed by the Balinese long-tailed macaques of Ubud (Bali, Indonesia)

Bite (BIT): To bring a stone to the mouth and place it between the teeth.

Comments: This pattern is typically performed using one or both hands, occasionally accompanied by foot support. Though most frequently performed by bringing a stone up to the mouth, this pattern may also occur when an individual brings their face down to a stone that is placed on a surface, though not when embedded in a substrate.

Carry (CAR): To hold or cradle a stone while moving from one place to another.

Comments: This pattern can be performed by using either the hands or mouth to grasp the stone. Stones are either held or cuddled against the body while the individual moves in a bipedal, tripedal, or quadrupedal manner.

Clack (CLK): To strike two stones, or a stone and an object, together with both hands moving in a symmetrical clapping gesture.

Comments: This pattern is performed utilizing either a precision or power grip, and always occurring while the stones are being held in front of the individual, away from the ground and other body parts.

Cover (COV): To lightly place an object upon or over a stone with the hands.

Comments: This pattern often resembles a peek-a-boo type of activity, where the stone is fully covered, and then frequently uncovered. Items regularly used to perform this activity are leaves, plastic, cloth, or dried grass. This pattern was not distinguished from "wrap" (WRP) in previous papers on Japanese macaques (Leca et al., 2007).

Cuddle (CUD): To grab a stone with the hand and hold it against a body part.

Comments: Body parts frequently utilized in this activity include the chest, abdomen, groin, and side of leg. This pattern can be performed with stones of various sizes, however frequently occurs with one large stone, or a series of smaller stones. Can be performed either on the ground, or away from the ground while held against the upper body.

Dislodge (DSL): To (potentially attempt to) remove a stone embedded in a substrate by scratching or rubbing it with the fingertips or mouth.

Comments: Stones are not always extracted from the substrate, however when they are, individuals either incorporate the newly unearthed stone(s) into the SH activities, or discard them immediately after dislodging. This pattern is most frequently performed on a dirt surface. When using the mouth to dislodge a stone, the teeth are used in place of the fingertips, moving in a similar motion.

Flint (FLN): To strike a stone held in one hand against another stone or object, that is held stationary in another body part.

Comments: Though this pattern is performed most frequently with the hands, utilizing either a power of precision grip, it is sometimes performed by using the mouth to hold the stationary stone (i.e., Flint in mouth). This pattern is always performed away from the ground.

Gather (GAT): To bring a stone to oneself, often collecting stones into a pile in front of oneself.

Comments: This pattern can be performed in a number of different ways, including picking motions, gathering large amounts of stones by sliding them towards oneself on the ground using the arms to guide them towards oneself, or the stacking of stones on top of one another. Stones can be gathered using both the hands and the feet. 
Grasp (GRP): To clutch a stone placed in front of or beside oneself, on the ground.

Comments: This pattern can be performed by both the hands or the feet, with a power grip, either tightly or loosely. This pattern frequently occurs while the individual's attention is elsewhere, or while another SH pattern is being performed.

Grasp-Walk (GRW): To clutch a stone in the palm of the hand while walking.

Comments: This pattern can be performed by either the hands or the feet, however it is most frequently held in the palm of the hand while the individual moves in a quadrupedal manner.

Groom (GRM): To pick at or scratch a stone with the fingertips.

Comments: Stones may be held or grasped in the hand or foot, or placed on the ground. This pattern resembles what would be observed during allo- or self-grooming sessions, however the actions are directed towards stones rather than a monkey's fur.

Hold (HLD): To pick up a stone and hold onto or clutch it for some time, keeping it away from both the body and other surfaces.

Comments: This pattern can be performed by both the hands and the feet, most frequently utilizing a power grip.

Lick (LIC): To bring a stone to the mouth and touch it with the tongue.

Comments: This pattern is typically performed using one or both hands, occasionally accompanied by foot support. This pattern is most frequently performed by bringing a stone to the face, rather than bringing the face down towards a stone that is placed on a substrate.

Move And Push/Pull (MAP): To clutch a stone that is placed on the ground with the arm(s) extended in front of oneself, and walk either forward or backward while the stone is rubbed against the ground.

Comments: This pattern can be performed using either one or both hands, and is sometimes performed in combination with a "bite" (BIT).

Move Inside Mouth (MIM): To insert a stone inside the mouth and move it with the tongue or the hands.

Comments: During this activity, the stone fully disappears inside the mouth. Stones can often be seen moving through cheek when performed.

Pick And Drop (PAD): To repeatedly take a hold of a stone with the hands and let it fall to the ground or into a cavity.

Comments: This pattern may be performed by picking up a stone and dropping it straight onto the ground, or the individual may let the stone roll down a body part, such as the arms, when the stone is being dropped. When using smaller stones, individuals frequently utilize a precision grip, and the action may resemble picking up small food items and quickly discarding them.

Pick Up (PUP): To take hold of a stone with one hand and place it into the other hand.

Comments: This action requires that the stone picked up be completely let go of by the original hand once placed into the open supporting hand. This action is performed most frequently when hands are placed in front of the body, away from any surface or other body parts.

Pound (PND): To strike a stone on the ground or an object, using a power grip.

Comments: Stones are typically pounded on a hard surface such as concrete or packed dirt. Most frequently utilized target objects are leaves, cloth, nuts or other stones. 
Pound-Drag (PDR): To strike a stone on the ground using a fluid motion and instantaneously drag the stone backwards once contact with the ground is made.

Comments: This pattern may resemble a "Pound" (PND) that is combined with a "Rub" (RUB), however, the pattern is performed without interruption as one fluid motion, and the latter rubbing portion of the pattern is interrupted as it does not include both a forward and backwards motion, only a backwards dragging motion of the hand.

Roll (ROL): To move a stone back and forth on a substrate in a rolling or rubbing motion, performed with a loose grip or open palms.

Comments: Though this pattern is most frequently performed with the hands, it is sometimes performed with the feet. This pattern resembles "Rub" (RUB), however the hand grip utilized for this activity is different.

Roll In Hands (RIH): To roll or rotate a stone back and forth in both hands, moving in an alternating sliding gesture, with a loose grip.

Comments: Stones are typically rolled along the length of the hand, utilizing the palms and fingers of both hands. This action can be performed either slowly or quickly. Stones are always held away from the ground or body when this pattern is performed.

Roll With Fingers (RWF): To move a stone back and forth on a substrate in a rolling motion using only the fingertips.

Comments: This pattern differs from "roll" (ROL) as only the fingertips are used to perform this pattern rather than utilizing the palm. A traditional grip is not utilized, rather the fingertips are pressed onto the stone with enough pressure as to guide the stone a short distance back and forth. This pattern is most frequently performed directly in front of the individual, using both hands to presumably stabilize and guide the stone. Stones used for this activity are very round.

Rub (RUB): To slide or move a stone back and forth on a substrate utilizing a power or precision grip.

Comments: Though this pattern may resemble "Roll" (ROL) the hand grip utilized in this activity is different. This pattern can be performed on the ground, or other substrates, such as on the fur of the individual performing the action (i.e., rub on fur). When a stone is rubbed on the individual performing the action, the duration is typically very short, and the stone is most frequently rubbed along the lower arms. Stones may also be used to groom other individuals. When used to groom other individuals, this pattern differs from "Groom" (GRM), as the focus is to rub the stone along the fur of an individual, potentially using it to assist in the grooming process, rather than to groom the stone itself.

Rub Together (RBT): To touch and move (in a rubbing motion) the surface of two stones together in an alternating sliding gesture.

Comments: This pattern is always performed with the hands placed in front of the individual, away from the ground and other body parts, utilizing either a power or precision grip.

Rub With Hands (RWH): To hold or grasp a stone with one hand (or foot) and move the palm of the other hand along the surface of the stone while applying firm pressure.

Comments: The hand performing the rubbing motion can either move back and forth along the surface of the stone(s), or perform the rubbing action in only one direction multiple times. Though this pattern most frequently occurs when stones are being held away from the ground or body, or in the water, it can also be performed when a stone is being grasped on the ground. 
Scatter (SCA): To disperse a stone with the hands in a scattering motion on a substrate, in front of oneself.

Comments: This pattern utilizes an open hand moving in a sweeping gesture across a substrate. This pattern differs from "gather" (GAT) as it does not bring stones towards the performer, rather moves them away, sweeping them to the side.

Shake In Hands (SIH): To move a stone in an open palmed hand by moving the hand back and forth, up and down, or repeatedly flexing fingers towards palm and back out again.

Comments: The placement of the hand in this activity may sometimes resemble "cuddle" (CUD) as the back of the hand can be held against or close to the body when performed. This pattern is always performed while the hand is away from the ground.

Shift In Hands (SFH): To completely transfer a stone from one hand to the other repeatedly, utilizing a cupping motion of the hands.

Comments: The entire hand is utilized in this activity as the curving of the fingers allows for the cupping motion required to completely pass the stone(s) off into the other hand each time. This pattern can be performed either slowly or quickly. Stones are always held away from the ground or body when this pattern is performed.

Slap (SLA): To hit a stone in a slapping motion with the palm or fingertips of the hand.

Comments: This pattern may resemble "tap" (TAP); however, it typically occurs one to few times, and is not used to hit or move a stone towards another stone, object, or body part. This pattern can be performed while a stone is being held, grasped, or on the ground, and can be performed with one or both hands.

Slap-Roll (SLR): To hit a stone in a slapping motion with the palm or fingertips of one hand, immediately followed by the rotation or rolling of the stone back and forth between both hands, moving in an alternating sliding gesture.

Comments: This pattern resembles a combination of two other SH patterns, namely a "slap" (SLA), followed by a "roll in hands" (RIH); however, it is performed only on the ground, rather than being held away from the ground or body while performing. This pattern is idiosyncratic, performed by only one individual.

Sniff (SNF): To bring a stone to the nose and smell it by inhaling.

Comments: This pattern is most frequently performed by bringing a stone to the nose using the hands, however it can also occur when an individual brings their face down to a stone that is placed on a substrate. The duration of this pattern is typically very short, however longer durations are sometimes achieved by performing repeatedly.

Tap (TAP): To move or tap a stone in a repeated sweeping gesture using the fingertips, against a substrate, object, or body part.

Comments: This pattern may resemble "slap" (SLA); however, it occurs multiple times, and the stone is tapped against another object, stone, or body part. Body parts most frequently involved include the hands, feet, tail, groin, and legs. This pattern can be performed in combination with objects and body parts (i.e., to tap a stone against a stone that is held by the foot) or just involving a body part (i.e., to tap a stone against the genital region, onto the side of the leg, or onto the other hand).

Toss And Catch (TAC): To lightly throw a stone upwards and catch it with one or both hands.

Comments: This pattern is idiosyncratic, only performed by one individual.

Toss-Walk (TSW): To lightly throw a stone, underhand, ahead of oneself while walking, then take hold of and clutch it in the palm of the hand.

Comments: This pattern differs from "throw" as it is not performed stationary, but while the individual is walking in a quadrupedal manner. The distance travelled by the stone is generally much shorter than with the pattern "throw," allowing the stone to be retrieved again after the tossing action occurs. 
Throw (THR): To toss a stone underhand, either in front of or behind the individual.

Comments: This pattern differs from the locomotive pattern "toss-walk" (TSW) as the individual generally performs this action while remaining stationary. Stones are thrown with the hands only, utilizing either one or both hands to perform the action.

Wrap (WRP): To encase or enclose a stone in an object, using the hands, either tightly or loosely, in what appears to be an attempt to bend or fold the object around the stone.

Comments: Items frequently used to wrap stones include leaves, cloth, plastic, and bundles of dried grass. This action can be performed either while the stone is placed on the ground, or while the stone is being held. The unwrapping of a stone that was previously wrapped with an object is also classified under this pattern.

Note. "Object" refers to a variety of objects other than stones, including vegetal materials and man-made items (e.g., wooden stick, leaves, grass, nuts, cloth, nylon rope, plastic bag, metallic rod; Pelletier et al., 2016). 